\title{
Perceptions of Pregnant Women Contributing to Late Initiation in Antenatal Care during the First Trimester in the Ubungo Municipal Council in Tanzania
}

\author{
Julieth Kristoamani, Gladys Reuben Mahiti
}

\section{ABSTRACT}

\begin{abstract}
Antenatal care serves as a key entry point for a pregnant woman to receive a broad range of services and should be initiated at the onset of pregnancy. The World Health Organization recommends for the first antenatal visits to be before 12 weeks of gestation. However, many pregnant women due to various reasons attend first antenatal care services very late and little information is known on why pregnant women delay initiating antenatal care clinics during the first trimester. This study aimed to explore the perceptions of pregnant women contributing to the late initiation of antenatal care during the first trimester Ubungo Municipal Council.
\end{abstract}

The study used purposive sampling to recruit thirty pregnant women for data collection through individual in-depth interviews. Pregnant women who attended antenatal care were recruited during antenatal care clinics and were interviewed in a room at the antenatal care unit. An in-depth interview was conducted with 30 pregnant women. Data were analyzed using thematic coding analysis.

The finding of this study indicates that, lack of awareness on the appropriate time to start antenatal care first visit, pregnancy as a normal health condition which does not require health personnel attention, late identification of the pregnancy, transport cost and service cost as a barrier due to low income, long waiting hours at the facility, lack of support from the spouse and men's working time table were major factors which pregnant women perceive to contribute late initiation in the antenatal care during the first trimester.

Results conclude that, community awareness-raising and training on the significance of early antenatal clinic (ANC) visits are needed. Stakeholders should also address factors affecting facility and service levels such as high service costs and long waiting times at healthcare facilities.

Keywords: Antenatal care, first trimester, late initiation, perceptions.
Published Online: December 11, 2020

ISSN: 2593-8339

DOI: $10.24018 /$ ejmed.2020.2.6.595

Julieth Kristoamani

Department of Development Studies, Muhimbili University of Health and Allied Science, Tanzania.

(e-mail: julietpm@yahoo.co.uk)

Gladys Reuben Mahiti *

Department of Development Studies,

Muhimbili University of Health and Allied

Science, Tanzania, Tanzania.

(e-mail: gmahiti2011@gmail.com)

*Corresponding Author

\section{INTRODUCTION}

Antenatal care is one of the strategies that have been found crucial for ensuring optimal care and good maternal and fetal health. It is estimated that 295,000 women died from preventable causes related to pregnancy and childbirth, while 94\% of these deaths occur in low and lower-middle-income countries [1]. Antenatal care is an opportunity to provide care for the prevention and management of existing and potential causes of maternal and newborn mortality and morbidity [2]. An early antenatal care visit allows for essential screening, testing, and treatment of genetic and congenital disorders that most effective early in the pregnancy. Additionally, the visit can potentially capture non-communicable diseases such as diabetes and guide on modifiable lifestyle risks such as smoking, alcohol consumption, drug abuse, obesity, malnutrition, and occupational exposures [3].

The World Health Organization (WHO) and Tanzania ministry of Health Community development, Gender Elderly and Children guidelines recommend a minimum of eight ANC visit, and the first contact is scheduled to take place in the first trimester (up to 12 weeks of gestation) [2]. However, studies show that in developing countries only $25 \%$ of pregnant women initiate their antenatal care first visit before 14 weeks gestation [4]. Recently data from Tanzania demographic survey shows that the proportions of antenatal visits have increased to $98 \%$, despite this increase, only $24 \%$ of pregnant women were reported to begin their antenatal care visit during the first trimester as recommended [5]. In Ubungo municipal council Only $10.3 \%$ of pregnant women initiate antenatal care during the first trimester [6]. Factors influencing the utilization of antenatal care have been widely studied. It has been shown that several factors hindered pregnant women to initiate early in antenatal care services such as economic status cause pregnant women to initiate late in antenatal care attendance for those with low economic status and those with a good economic status they tend to initiate early [7]. Long waiting hours into health care facilities 
and informal payments were the barriers that influence the late utilization of antenatal care services [8]. Other studies show that experience with a previous pregnancy and lack of a spouse may facilitate early or late initiation in ANC [9].

Although these studies provide insights into factors for late attendance of ANC, few studies employ qualitative methodology in Tanzania. However, there is little information on why women delay antenatal care in the first trimester in Tanzania among pregnant women in Ubungo Municipal Council. Therefore, this study focused on exploring perceptions of pregnant women contributing to late initiation in antenatal care during the first trimester, this will help the decision-makers to be informed, design, and implement effective interventions to address this critical public health issue.

\section{METHODS}

\section{A. Study Design}

The study was exploratory using a qualitative approach. The qualitative approach is flexible and allowing adjustment of new information during data collection and this helped data saturation that helped in the development of themes [10].

\section{B. Study Setting}

The study was conducted in Sinza Palestina hospital, located in the Ubungo Municipal council, Dar- es- Salaam region in Tanzania. Sinza Palestina hospital is functioning as a District hospital, public-owned health facility in Ubungo municipal council. Ubungo Municipal council is an urban area with approximately $1,043,549$ populations. The council has 14 wards, and 91 streets and covers a total surface area of $210 \mathrm{~km}^{2}$. The council has a total of 135 functions health facilities where 21 of them are public-owned health facilities. Out of 135 facilities 58 health facilities provide reproductive and child health services of which 21 health facilities are public-owned.

\section{Sample Size and Sampling Technique}

The study sample enrolled thirty pregnant women. Participants were selected through purposive sampling. They were asked some critical questions like the number of weeks of pregnancy, including demographic data, to determine their interview eligibility. The investigators informed the pregnant mothers attending antenatal care clinics about the study by service providers at the antenatal care unit during antenatal care services. Information about the study's voluntariness was given, and those who wished to participate were referred to the researcher assistant for interviews in a room in the clinic.

\section{Data Collection Procedure}

Data collection was done through in-depth interviews. An in-depth interview is a data collection tool that ensures, the same information is collected from all participants, systematically and comprehensively [11]. Interviews were conducted face to face using an interview guide. The interview guide was translated from English into Kiswahili and back to English to ensure the validity of findings. Probing was an important tool to ensure the credibility or true value of the data, as it allowed for the clarification of interesting and relevant issues raised by the respondent. The interviews were audio-recorded. Written notes were also used to record information as an addition to the audio recorded. Data collection was stopped once saturation was reached. Saturation has emerged as a Gold standard technique in qualitative research which involves continuing sampling the study participants, until when now new data or no new themes/no new code is generated from the participants. This means, the generated information has reached the saturation point, where more information becomes irrelevant. At this point, the researcher is required to stop the interview process [11]. Each interview took between thirty to forty-five minutes and was assigned a code and a date to maintain confidentiality. The assistance researcher's diary notes were collected and analyzed at the end of each day to ensure proper judgment.

\section{E. Trustworthiness of the Study}

Trustworthiness in a qualitative study aimed to support the argument that examines findings is worth paying attention to. It is the degree of confidence in data, interpretation, and methods used to ensure the quality of a study [12]. The trustworthiness was based on five dimensions, credibility, dependability, conformability transferability, and authenticity [12].

Credibility refers to confidence in the truth that the data and the interpretation thereof are a true reflection of the participants' experiences, views, and beliefs [13]. The effort was made to ensure that the selected participants possess the same experiences of booking early at first trimester and late means after the first trimester for first antenatal care visits. Only participants who met the selection criteria were purposively sampled. To ensure credibility, member checking was taken into consideration.

A member check is a crucial process that any qualitative researcher should undergo because it is the heart of credibility [12]. In this study, the Recording of respondent's interview was used to ensure that no distortions or missing data, this helped to eliminate researcher bias when analyzing and interpreting the results

Dependability refers to the stability of data even if the work were to be repeated in the same context, with the same method and the same participants [13]. In this study, dependability was ensured by providing a clear description of the process.

Conformability ensures that the work of findings is the result of the experiences and ideas of the participants, rather than the characteristics and preferences of the researcher, to ensure conformability in this study, a detailed methodological description and data were allowed to speak for themselves [13]. To ensure that no bias influences the results, tape recordings, and field notes were utilized and kept for further auditing; quotes from the informants were also included.

Transferability refers to the potential for extrapolation, it relies on the reasoning that findings can be generalized or transferred to other settings of groups [13]. In this study, transferability was taken care of by a proper description of the study context.

Authenticity refers to the extent to which fairness and faithfulness are displayed by the researcher in different realities [14]. Authenticity is evident when the report describes the feelings of the participant's lived experiences. 
The audio recording and transcripts were available to be seen by the supervisors. All participants' signed consent forms were kept safe in case proof was required.

\section{F. Data Analysis}

Data were analyzed using a thematic analysis approach, the thematic analysis focused on examining themes within data, the process and analysis followed the following steps [15].

The thematic analysis provided a highly flexible approach that can be modified for the needs of many studies, providing a rich and detailed, yet complex account of data. It offers a more accessible form of analysis, particularly for those early in their research career [11]. It is also a useful method for examining the perspectives of different research participants, highlighting similarities and differences, and generating unanticipated insights. Thematic analysis is also useful for summarizing key features of a large data set, as it forces the researcher to take a well-structured approach to handle data and helping to produce a clear and organized final report [16]. The thematic analysis process started with familiarization with the data, generating initial codes, integration of codes into descriptive themes, synthesis of descriptive themes into analytical themes, and producing the report

TABLE1: AN EXAMPLE OF ANALYSIS INDICATING CODING OF TEXT DATA AND DEVELOPMENT OF THEME

\begin{tabular}{|c|c|c|c|}
\hline Text & Code & $\begin{array}{c}\text { Descriptive } \\
\text { theme }\end{array}$ & $\begin{array}{l}\text { Analytical } \\
\text { theme }\end{array}$ \\
\hline $\begin{array}{l}\text { "The reason why I } \\
\text { started antenatal } \\
\text { care (ANC) clinic } \\
\text { late is due to the cost } \\
\text { of some tests. I was } \\
\text { thinking about the } \\
\text { expenses for the } \\
\text { blood test and } \\
\text { ultrasound. Since I } \\
\text { don'thave moneyfor } \\
\text { meals" }\end{array}$ & $\begin{array}{l}\text { - Start } \\
\text { antenatal } \\
\text { care late } \\
\text { - Expenses } \\
\text { for blood test } \\
\text { and } \\
\text { ultrasound } \\
\text { - Lack of } \\
\text { money for } \\
\text { meals }\end{array}$ & $\begin{array}{l}\text { - Pregnant } \\
\text { mothers lack } \\
\text { money to start } \\
\text { antenatal care }\end{array}$ & $\begin{array}{l}\text { - Financial } \\
\text { difficulties } \\
\text { among } \\
\text { pregnant } \\
\text { women as a } \\
\text { barrier to the } \\
\text { initiation in } \\
\text { antenatal care } \\
\text { services during } \\
\text { the first } \\
\text { trimester }\end{array}$ \\
\hline
\end{tabular}

\section{G. Ethical Considerations}

The study was approved by the Institutional Review Board of the Muhimbili University of Health and Allied Sciences with reference number DA. 287/298/01A. Permission was solicited from the Municipal director and District Medical officer together with the facility in charge before data collection was initiated. Individual participants gave informed consent to participate and were also assured of confidentiality. Data for this study were saved in a passwordprotected computer to avoid access from unauthorized personnel.

\section{RESULTS}

A total of 30 pregnant women were interviewed. Their ages ranged from 16 to 35 years. Approximately 16(53.3\%) pregnant women were aged 25 and below and 14(46.7\%) participants were above 25 years. Twelve pregnant women of those interviewed for this study had a secondary education level. Twenty-three participants reported having attended the late first antenatal care visit, twenty-six were married, and seventeen of them were in para one.

\section{A. Inadequate Knowledge of Couples on Antenatal} Services in the First Trimester

Most pregnant women especially during first pregnancy delayed antenatal care (ANC) attendance because they were not aware when to start antenatal care services clinics and the number of visits recommended. One participant has this to say,

"It is due to the situation people said that ANC not supposed to start early, it should be delayed and go after four or three months". (IDI with pregnant women)

According to our traditions and customs, men are the ones who had decision making power on where and when women should seek health care services. Therefore, if men do not have enough knowledge on the timing of initiating antenatal care make it difficult to influence pregnant women to initiate the antenatal care service at the right time. One respondent illustrates this way,

"It is not easy for my partner to understand even if I tell him. In his understanding, he is saying the clinic is from the second trimester. I forced to come in the first trimester and he was not ready." (IDI with pregnant mother).

The majority of pregnant women considered pregnancy to be a normal life event rather than a condition that requires the attention of health personnel. Some of the pregnant women said they would seek antenatal care only if they felt unwell.

"I said I will start ANC if the pregnancy starts to bother me”. (IDI with Pregnant mother).

Some pregnant women had not known they were pregnant for weeks or sometimes months because it is the first time for them to have a pregnancy. This situation makes them delay in accessing care early

"This is my first pregnancy so, I could not realize early that is why I was a late attendant in my first antenatal care clinic”. (IDI with Pregnant mother).

B. Financial Difficulties among Pregnant Women as a Barrier to the Initiation in Antenatal Care Services during the First Trimester

In Tanzania antenatal care is supposed to be provided for free, pregnant women with low-income status experienced difficulty when told to pay. Few pregnant women reported some cost that required to be paid for the service of some investigation of blood samples and ultrasound. This situation leads them to delay starting antenatal care clinics as recommended. One participant said this way.

"The reason why I started antenatal care (ANC) clinic late is due to the cost of some tests. I was thinking about the expenses for the blood test and ultrasound. Since I don't have money for meals". (IDI with Pregnant mother) Few participants reported that transportation cost to and from the clinic is a barrier that makes pregnant women delayed initiating early in antenatal care services. Transport cost depends on the distance from the health care facility. Those who lived far from the facility paid more cost than those who lived near the facility. One participant was quoted

"Transportation cost can also be a reason to be late to start an antenatal care clinic in the first trimester because it depends on where the person comes from. Because someone who comes to the clinic and uses $5000 \mathrm{TzS}$, is quite different from the one who uses 2000 Tsh. Remember people's affordability varies." (IDI Pregnant 
mother).

C. Antenatal Care Policy and the Health System as a Barrier Contributing to Late Initiation in the Antenatal Care Services during the First Trimester.

During the first visit, the antenatal care policy requires every pregnant woman to conduct the different test to know the health status of the mother for the well being of the unborn child. Some participants said that long waiting hours at the clinic due to different health tests is a barrier that makes them attend late in the first visit. This was supported by one pregnant woman, who said that:

"Test results delayed, for example, it takes about ten hours to wait for the blood and ultrasound results, I didn't receive my results until I went into the medical officer in charge to complain about the delay of the results and in that day I failed to get the clinic services" (IDI with Pregnant mother).

Respondents reported that felt reluctant to start antenatal attendance because their partners/spouse did not have time to accompany them in the antenatal care clinic due to the working timetable does not support them to get a chance in the weekdays.

"I started my antenatal clinic late, I wished to get the chance to come with my partner but I failed because he was not getting even a little chance to come home during the weekdays because he usually comes in the weekends, which is also very rare because of his working time table." (IDI with Pregnant mother).

\section{DISCUSSION}

The study aims to explore the contributors to poor antenatal care attendance in the first trimester among pregnant women in Dar- es- salaam Tanzania, our findings show that inadequate knowledge in couples on when and importance of utilization of Antenatal care services, economic status, and antenatal policy were the major contributors to poor antenatal care attendance in the first trimester.

A major finding of the study was the lack of knowledge on the importance of antenatal care clinics. They do not understand that antenatal care is primarily provided to detect or treat diseases. That is why many participants said they did not have any problems that required them to go early into the antenatal care clinic or need the intervention of health personnel. Some respondents assumed that there were no benefits in booking in the first trimester. There is a perception that pregnant women view pregnancy as normal health conditions, and it does not require any attention of health personnel unless a woman felt unwell it is a time to seek healthcare. Therefore, pregnant women and the community at large perceived that antenatal care is for curative rather than a preventive intervention. This is in line with a study done in Cameroon which reported that most women start antenatal care late because of the belief that there are no advantages to start antenatal care in the first trimester [17].

Other participants said they were advised by their spouses/partners to initiate antenatal care after the second trimester. The study reveals that the spouse/ partner has an important role to play in deciding where and at what time his wife is required to initiate the antenatal care services. Therefore, there is a need to develop health education programs that empower spouses and other community members to improve their knowledge of the importance of early antenatal care services. This is in line with a study conducted in southwestern Nigeria, incorrect advice on the best time to start antenatal care from relatives or partners was highlighted as one of the reasons why women started antenatal care late [7].

Financial constraints such as transportation costs; costs for antenatal care were seen as important barriers for pregnant women to initiate antenatal care services during the first trimester. Though it has been officially announced in Tanzania that maternal and child health care is free services, there are some costs for the service in the clinic such as blood and ultrasound test; these tests are not provided free, thus perpetuating late antenatal care booking. This finding is similar to other studies conducted in Nigeria and Southern east Tanzania which reported that the costs of obstetrical care determine whether the mother will attend antenatal care in the first trimester [18], [19]. Among the proposed approach to increase community, health coverage is to ensure that every person has health insurance. A study conducted in Rwanda in 2014 reported that having enrolled in public health insurance is associated with early antenatal care initiation [20]. Our findings show that for the pregnant women to be enrolled in antenatal care clinic early there is a need to persuade and ensure that all pregnant women have a health insurance card

It was indicated by women in this study that lack of fares for transportation is a barrier for them to register early in antenatal care services. The transportation cost to and from the health care facility prevented women from going for antenatal care visits and even when fares were at a low rate, women who lived in extreme poverty could not afford to pay. These findings are supported by the previous study done in Nigeria, which reported that the utilization of antenatal care is influenced by economic factors such as costs related to the distance to health care facilities such as transportation costs [7].

Another factor that contributes to late initiation of antenatal care during the first trimester is long waiting time has also a negative impact on the decision to start antenatal care clinic early. Also, long waiting time was one of the determinants of the low utilization of antenatal care clinics during the first trimester. The findings of this study are similar to the study done in Guinea, which revealed that that long waiting time is the reasons for this could be that, when pregnant women anticipate, the hours, she expects to wait until getting antenatal care services, she delays initiate early at firsttrimester antenatal care visit [8]. Corresponding to the result of this study, a study conducted in eastern cape, south Africa in 2018 shows that lack of health care personnel is often a barrier to effective antenatal care services [21]. Therefore, the findings of this study suggest increasing the number of health care personnel in health facilities this will help to reduce a long waiting time at the health facility.

Antenatal care and HIV testing guidelines encourage the escort of a spouse during the first antenatal care visit. The aim of this is to enable health care workers to provide health education to the couple on danger signs during pregnancy, 
individual birth preparation, and more important counseling and testing for STI and HIV. Therefore, to achieve this health care provider is abusing this influence by imposing minor rules that a pregnant woman must come with her partner on the first visit. Pregnant women who are not able to come with their partners were required to get an introductory letter from the street leaders otherwise they may not get antenatal care services. Respondents reported that failed to get an escort from her partner/ spouse of her working timetable makes him fail to get a chance to escort his wife in antenatal care clinic which makes pregnant women start antenatal care attendance late because they are worried that they would be denied getting antenatal care services. These findings contrast with the study conducted in Dar- es Salaam region in Tanzania which found that antenatal care policy act as a barrier for early antenatal care attendance due to pregnant women who had challenges to get a male partner's support for different reasons felt reluctant to start antenatal attendance [9]. It has been a long time in society there has been a perception that pregnancy and child health have been considered as women's issues, so it should not be mandatory for a pregnant woman to come with her partner. Therefore, when women came alone, they should get all the required antennal care services.

The interpretation of the spouse policy should not be left to the health care providers who have a view of achieving health-related goals such as increasing the number of couples testing for HIV where this leads to ignoring the social consequences of the policy. Therefore, this requires regional and district health officials to provide mentorship to health care providers on the proper interpretation of the policy and proper way of implantation of this policy and focus on the contents. Findings suggest that community members especially men should be sensitized on the importance of timing of antenatal care and HIV testing during pregnancy.

\section{CONCLUSION AND RECOMMENDATIONS}

In conclusion, women in Ubungo Municipal council have provided information on the various aspects of late antenatal attendance at Sinza Palestina hospital in the Ubungo Municipal such as Inadequate Knowledge Of Couples On Antenatal Services In The First Trimester, Financial difficulties among pregnant women as a barrier to the initiation in antenatal care services during the first trimester and antenatal care policy and the health system as a barrier contributing to late initiation in the antenatal care services during the first trimester. Therefore, the following recommendations if implemented may improve the timely accessing of antenatal care services among pregnant women.

1. Continuous health education on when and the importance of early antennal care visits should be provided by the Government through mass media and community sensitization meetings and in the clinic, where pregnant women attend.

2. The government should increase more health care facilities and an increasing number of health care workers to expand the provision of antenatal care services near to its population to reduce geographical and indirect costs associated with antenatal care services attendance.
3. District Medical Offices should increase accessibility of antenatal care clinic (ANC) services by proving -scheduled outreach programs in remote areas

4. The community should be sensitized to join in improved Community health fund (iCHF) this will help to cover service costs, especially for pregnant women.

5 . Women should be encouraged to engage in economic activities to supplement their income.

\section{ACKNOWLEDGMENT}

We would like to thank Ubungo Municipal director and in charges of the the district and health facilities for approval to conduct the study. Also, our special thanks are directed to pregnant women for their cooperation especially the provision of essential information which aided the successful completion of this study. We would like to acknowledge all colleagues at Muhimbili University who gave their comments during preliminary stages of manuscript writing.

\section{REFERENCES}

[1] Mortality T. TRENDS IN 2000 to 2017 TRENDS IN MATERNAL MORTALITY : 2000 TO 2017. WHO, UNICEF, UNFPA, World Bank Group and the United Nations Population Division. 2017.

[2] WHO. World Health Organization recommendations on antenatal care for a positive pregnancy experience. World Health Organization Geneva, Swizerland. 2016.

[3] EBCOG Scientific Committee. The public health importance of antenatal care. Facts, views Vis ObGyn. 2015.

[4] Moller AB, Petzold M, Chou D, Say L. Early antenatal care visit: a systematic analysis of regional and global levels and trends of coverage from 1990 to 2013. Lancet Glob Heal. 2017.

[5] MoHCDGEC. Tanzania Demographic and Health Survey and Malaria Indicator Survey (TDHS-MIS) 2015-16. Dar es Salaam, Tanzania, and Rockville, Maryland USA. 2016.

[6] District Health profile Ubungo MC , 2018.

[7] Adekanle DA, Isawumi AI. Late antenatal care booking and its predictors among pregnant women in South Western Nigeria. Online J Heal Allied Sci. 2008.

[8] Andrew EVW, Pell C, Angwin A, Auwun A, Daniels J, Mueller I, et al. Factors affecting attendance at and timing of formal antenatal care: Results from a qualitative study in Madang, Papua New Guinea. PLoS One. 2014.

[9] Mgata S, Maluka SO. Factors for late initiation of antenatal care in Dar es Salaam, Tanzania: A qualitative study. BMC Pregnancy Childbirth. 2019.

[10] Sutton J, Austin Z. Qualitative research: Data collection, analysis, and management. Can J Hosp Pharm. 2015.

[11] Braun V, Clarke V. Qualitative Research in Psychology Using thematic analysis in psychology Using thematic analysis in psychology. Qual Res Psychol. 2006.

[12] Polit DF BC. Qualitative Descriptive Studies. In: Essentials of Nursing Research: Appraising Evidence for Nursing Practice 8. 2014

[13] Guba E, Lincoln Y. Establishing Trustworthiness. In: Naturalistic Inquiry. 1985.

[14] Polit DF, Beck CT. Analyzin qualitative data. In: Nursing research generating and assessing evidence for nursing practice. 2008.

[15] Alhojailan M. Thematic Analysis: A critical review of its its processes and evaluation. West East J Soc Sci. 2012;

[16] King N, Cassell C, Symon G. Using templates in the thematic analysis of texts. Essent Guid to Qual methods Organ Res. 2004.

[17] Warri D, George A. Perceptions of pregnant women of reasons for late initiation of antenatal care: A qualitative interview study. BMC Pregnancy Childbirth. 2020.

[18] Ada CN, Ifeoma N, Ijeoma LO, Okwudili E, Umebuani N. Determinants of late booking for antenatal care among pregnant women in selected hospitals in South East Nigeria. Int J Nurs Midwifery. 2018.

[19] K. G, S. A, T.R. G, J.A. S, B. O. Timing of antenatal care for adolescent and adult pregnant women in south-eastern Tanzania. BMC Pregnancy and Childbirth. 2012.

[20] A. M, F. M, F. M, L. B, F. S, D.R. T, et al. Assessing predictors of 
delayed antenatal care visits in Rwanda: A secondary analysis of Rwanda demographic and health survey 2010. BMC Pregnancy Childbirth. 2014.

[21] Kaswa R, Rupesinghe GFD, Longo-Mbenza B. Exploring the pregnant women's perspective of late booking of antenatal care services at Mbekweni Health Centre in Eastern Cape, South Africa. African J Prim Heal care Fam Med. 2018. 